\title{
User Participation in the Process of Creating a Citizen Science Portal
}

\author{
Andreas Divanis $^{\mathrm{a}}$ (corresponding author), Alissa Lüpke ${ }^{\mathrm{b}}$ and Liqiu Menga \\ andreas.divanis@tum.de, alissa.luepke@tum.de, liqiu.meng@tum.de \\ ${ }^{\mathrm{a}}$ Chair of Cartography, Technical University of Munich, Munich, Germany \\ brofessorship of Ecoclimatology, Technical University of Munich, Munich, Germany
}

\begin{abstract}
Citizen science (CS) relies on cooperation with members of the public. This usually requires a lot of contact to reconcile the needs of the researchers and project staff with the user to retain an active user base. We explore with our CS web portal the potential of including the participants in the creation and design process of the portal. This is done by providing the users with easy access to our design and research staff and approaching people for qualitative feedback. To a large extent, our users do not have obvious ties to academic institutions, however, the vast majority of feedback comes from academics. We categorized the feedback, explored users' backgrounds, and compared our active user retention to another CS project, which turns out to be comparable.
\end{abstract}

Keywords: citizen science, web portal, web map, user experience, participatory research

\section{Introduction}

Citizen science projects rely on public participation to gather scientific data (Bonney et al., 2009). The idea to involve citizen scientists in the development and design of projects is not new (Wiggins and Crowston, 2011). The participants may not be experts in the project domain. For the project to be successful, the underlying intent and the process of data collection must be clearly communicated to participants. Face-to-face interaction between project members and participants often helps to improve data quality (Rüfenacht et al., 2021). Nevertheless, in situations where this is not easily possible, such as during the Covid-19 pandemic with meeting restrictions, citizen science projects have to shift the focus to the development of an online user base. This requires developers to invest more effort in understanding the online experience and behavior of participants in order to adequately communicate the design intent.
In this work, we examine our approach to include users of our climate research portal in the design during its creation stage. Since we follow an open data policy, all the collected data is available for users to download. Users may also benefit from interactive mapping and analytics tools on our web portal, such as risk mapping for allergenic species, interactive choropleth mapping, filtering by variables, and geostatistical computing.

The interactive tools have made our web portal attractive for teachers who in turn may recruit their students as further users. 55 schools in Bavaria have already endorsed participation in our project as listed under https://www.portal.baysics.de/for-students.

However, it is quite a challenge to impose mapping and analytics tools on new users who work with many different mobile devices and thus face different constraints when using our portal. Furthermore, portal developers are experts in various domains who tend to take it for granted that the design is easy to understand and shares common rules with other software, such as GIS tools, Statistical Analysis tools, or prevailing web portals. This assumption may not hold for average users.

In order to engage existing users in the process of creating our portal and to attract new users, thus benefit from their knowledgeable suggestions on what issues are important and what features should be added, we need to align the online experience of developers and users with the portal design.

In section two we introduce the background of our project and how users are supposed to interact with the portal from the designer's perspective. Our approach of how to engage participants is described in section three with results presented in section four and conclusions in section five. 


\section{Background}

The project BAYSICS (Bavarian Synthesis Information Citizen Science Portal for Climate Research and Science Communication) funded by the Bavarian State Ministry of Science and Art (https://www.bayklif.de/en/startseite-en/) is a cluster bundling 10 subprojects. It has the objective to tackle common questions of climate research including the different perceptions of users who see climate change as a distant phenomenon or who see the data and science behind it as opaque or even untrustworthy. Policies to adapt to climate change must be seen by citizens as legitimate, acceptable, and feasible. A useful measure is to make scenarios of climate change more visible to citizens by having them collect data related to climate change and explain the background. For example, allergy sufferers might notice how their symptoms of pollen allergy start at different times due to climate change. Other users might notice while collecting elevation of tree species how certain tree species grow at a different altitude in the Bavarian Alps than historical records.

On the BAYSICS Portal and its corresponding progressive web app as shown in Fig.1, users in Bavaria may contribute their observations to an open data pool by adding them to a data collection while simultaneously using analysis tools to visualize and interpret the data.

\section{Figure 1: Map view of the BAYSICS portal}

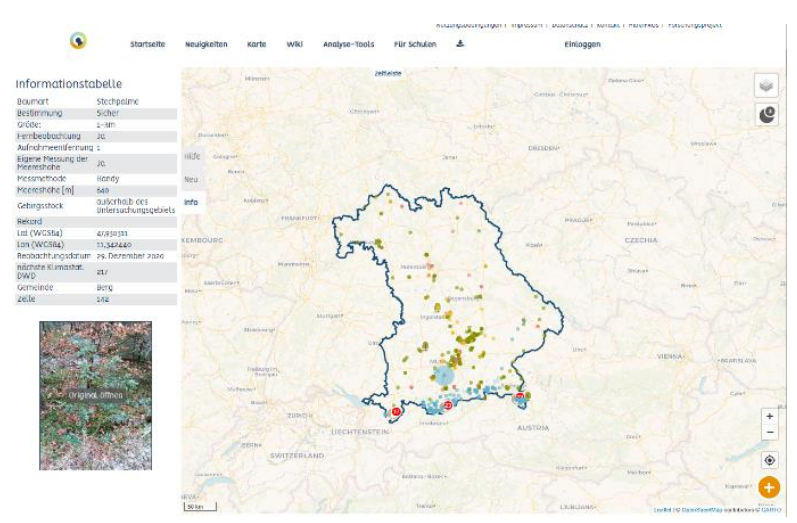

Our project members are interested in four different data categories that citizen users may help collect: (1) the yearly phenological development stages of plants, which are sensitive to temperature (Hatfield and Pruger, 2015) and atmospheric conditions (Menzel et al. 2020); (2) allergenic species, as pollen drift in the air is also influenced by temperature and atmospheric conditions (Ziello et al., 2021); (3) the elevation that tree species grow in alpine areas and whether they break the historical records (Rösler et al.2020); and (4) animal observations in urban areas that serve as testing sites as the conditions in cities often differ from that outside.

Our efforts to draw users' attention to the portal included outreach work targeting schools, environmental associations, hiking associations, and interviews with local news outlets. Moreover, the content of our portal differs from previous portals in Bavaria. While some portals with plant or animal data already exist (such as https://www.igel-in-bayern.de/, which records hedgehogs), we collect more species, more categories, and make this data available for free download for every user. Another measure to attract users is that we offer built-in data processing functions that are not available in comparable portals in Bavaria, making it more attractive to teachers and students. With those features, we aim to include the users in the scientific process of data analysis. We plan to incentivize user participation by acknowledging them in publications based on their contribution if they choose to disclose their name.

At the time of writing this paper, the data collected from users contained 589 entries for the phenological category, 237 entries in the category of allergenic species, 436 entries in the category of elevation of trees, and 131 entries in the category of animals. Data is stored in a relational database, with each row containing a location, date, category-specific attributes (such as elevation, flowering time, plant size, photo link, and associated photo), and attributes for quality assessment (such as distance from an observed object, method or device used to enter the location, a comparison to elevation data by the Bavarian State Office for Survey and Geoinformation, and if the user is confident in the species recorded). The data is saved as a table with point geometries in a PostGIS database an users can download the data as CSV files.

As portal developers, we tried to design the input forms for creating new entries easily comprehensible and intuitive to operate. The feedback from the users showed that this was one of the areas we profited the most from user-proposed changes.

\section{Our approach}

\subsection{User participation in the design}

Participation of the users in the design part of our project took place in the beginning mainly in two ways. In the first way, one of our experts acted as a visible contact person to receive suggestions sent by users. Upon using 
our portal or our website, users became aware that the the portal was in the design phase. With this notice, users were invited directly on the landing page to contact us with their suggestions. Our contact person then notified the relevant project member involved in the creation of the feature or if necessary, established direct contact between the user and the project member. The second way was more straightforward. We visited potential citizen participants who were not yet involved in the portal, exposing them to the portal or application for the first time, asking them to provide some feedback, and documenting their ideas and difficulties.

\subsection{Categorization of users' suggestions}

One of the key points of aligning users' suggestions with our vision was to judge from which suggestion we would benefit. However, as project member, we could hardly give an objective view on which suggestion is more beneficial than other suggestions. Therefore, we decided to rank suggestions according to the difficulty of their implementation. If a suggestion was not too difficult to implement or conflicted with other suggestions, it was implemented. Meanwhile, we reserved the right to prioritize the suggestions that we deemed most relevant to our ongoing research.

We ordered feedback by six categories:

1. keep an option

2. correct unintended system behavior

3. easy to implement and relevant to research

4. easy to implement but not relevant to research

5. hard to implement but relevant to research

6. too hard to implement or detrimental

Category from one to five is what we tried to keep when faced with multiple suggestions at the same time. Suggestions that fell into category 6 were discarded. Here are some paraphrased examples from each category:

Category 1: Users asked to keep the option to switch between different background maps, as they took a very bright map to contrast data points, a topographic map to plan their routes for entering new observation, and an aerial image to cross-check if they entered a tree at the correct location. We marked this kind of behavior as to keep the option.

Category 2: Users experienced unintended system response when uploading photos on their device, possibly due to an unspecified file format. Also, they alerted us of the GPS inaccuracy at the border of Bavaria that caused their entries to be wrongly assessed as being outside the border. The unintended system behavior was the main source of users' frustration, therefore, it must be removed with a high priority.

Category 3: Users asked us to display the exact percentage on the leaf-fall sliders for plants in the input form as they had difficulties estimating it from a slider that only showed a minimum and a maximum.

Category 4: Users asked us to change the color of the example text in the pre-filled input form as it was hard for them to distinguish between the pre-filled text and the text they wrote on their device.

Category 5: Users asked us to implement a function that allows them to enter observations with an unreliable internet connection or while offline, as well as giving them options to have their usernames rated for observations.

Category 6: Users asked us to make the web portal run on their windows phone and to support an old version of internet explorer. Other users asked us to integrate AI functions for the automatic recognition of species captured with their cameras. In addition to technical difficulties of implementation, we were also aware that some user suggestions are contradictory, e.g., some users preferred small symbols on the maps while other users required bigger symbols so as they could easily click on them.

\subsection{Evaluation of the suggestions}

Some of the users' feedback was domain-specific and suggested a lot of familiarity with web maps, visualizations, and user design in general.

This posed an interesting task to us to find out how many users sending feedback might have an academic background, thus with a higher likelihood of familiarity with tools from our portal. We approached this by looking at the domain of the registering emails, to see how many were associated with universities and research centers. Participating users need to sign up with an email address, but no other information is required, as we wanted to keep it as accessible as possible. The email domains after the @-symbol were gathered and grouped. This resulted in 105 unique domains, which we manually curated to see if they belong to an academic institution.

By considering users with such a domain being related to academic background, we could estimate that about seventy percent of all suggestions to our portal were 
given by people with academic backgrounds as shown in table 1.

\section{Table 1: Suggestions by users}

academic implemented not implemented

\begin{tabular}{rrrr}
\hline 1 & yes & 24 & 6 \\
2 & no & 11 & 6
\end{tabular}

As of the date when generating this table, we counted 438 users among which 344 were deemed to be active based on their last login date within the last 100 days. Twitter defines inactivity by login date (https://help.twitter.com/en/rules-and-policies/inactivetwitter-accounts) of 6 months or earlier (https://www.bbc.com/news/technology-50567751).

Since we have a much smaller user base, half of that time is reasonable for our case. In addition, our portal has a strong focus on outdoor plants, we expect at least some hiatus of users during the winter.

Of the 344 active users, only 46 had an academic email domain, who however made ca. $70 \%$ of suggestions. Furthermore, suggestions that fell in categories three and five, which we deemed relevant to our research, came mostly from people with ties to an academic email domain (Table 2).

Table 2: Suggestions by categories

\section{category academic non academic implemented}

\begin{tabular}{rrrr}
\hline 1 & 4 & 3 & 7 \\
2 & 1 & 2 & 3 \\
3 & 11 & 2 & 11 \\
4 & 3 & 0 & 2 \\
5 & 10 & 5 & 13 \\
6 & 1 & 5 & 0
\end{tabular}

\section{Results}

With regard to user participation over time (Fig. 2) we observed a slow increase of users before March 2021 and a sharp growth since then.

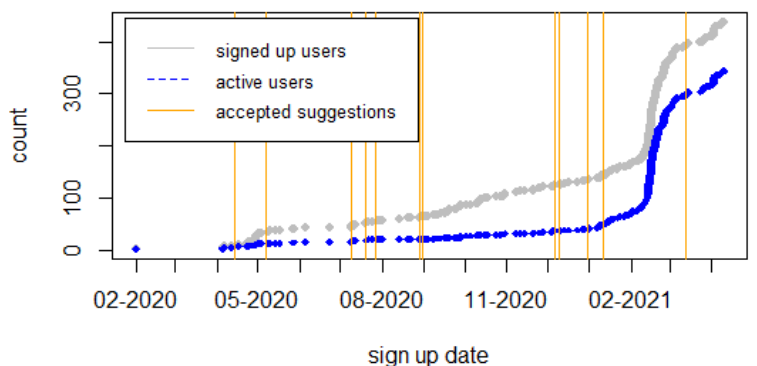

Figure 2: Portal users

The date of the suggestions does not seem to correspond to the growing user count, which is not unexpected as we presumed the user participation through suggestions to be a way to keep users interested, instead of attracting new users. The strong increase of users since March 2021 coincides with the start of our advertisement and outreach work, but not necessarily with the more favorable weather conditions, which seem to be most effective for the expansion of the user base. The largest disparity between signed-up users and active users from November 2020 to January 2021 seems to coincide with the pandemic measure phases in Bavaria.

\section{Conclusion}

We did not find that involving users in the creation process leads to a growth in users, however, we managed to keep a healthy active user count despite the disadvantageous situation of the winter season combined with the ongoing pandemic.

Monitoring participants' needs is necessary in order to retain active users, which is normally realized by meeting the participants in person (West and Pateman, 2016). Although it is still too early to conclude that the involvement of participants in our portal design is a good practice, we have retained an active user count that is comparable or better than other citizen science projects such as project Evolution MegaLab where 38\% of users actively participated (Worthington et al.,2012).

While a web portal has the potential to reach many more people than a face-to-face campaign with interested citizen scientists, its communication with users is less straightforward. On the other hand, it forces developers and researchers to be as unambiguously as possible in phrasing and designing since you cannot explain things quickly in a half-sentence.

The fact that the majority of our suggestions came from users with ties to academia may sound suboptimal 
because our goal is to attract normal citizens to influence our design choices. Since our portal is a work in progress and still in its construction phase, the preliminary findings will guide us to strengthen our effort of approaching users with no ties to academia and understanding their requirements.

\section{References}

Bonney, R., Cooper, C. B., Dickinson, J., Kelling, S., Phillips, T., Rosenberg, K. V., \& Shirk, J.: Citizen Science: A Developing Tool for Expanding Science Knowledge and Scientific Literacy. BioScience, 59(11), 977-984, https://doi.org/10.1525/bio.2009.59.11.9, 2009.

Hatfield, J. L. and Prueger, J. H.: Temperature extremes: Effect on plant growth and development, Weather and Climate Extremes, Volume 10, Part A,2015, 4-10, https://doi.org/10.1016/j.wace.2015.08.001, 2015.

Menzel, A., Yuan, Y., Matiu, M., Sparks, T., Scheifinger, H., Gehrig, R. and Estrella, N.: Climate change fingerprints in recent European plant phenology, https://doi.org/10.1111/gcb.15000, 2020.

Rösler, S., Olleck, M. and Ewald, J.: Klimaforschung auf Otto Sendtners Spuren - mit Citizen Science die Baumgrenzen in den Bayerischen Alpen untersuchen, Jahrbuch des Vereins zum Schutz der Bergwelt (München), 85. Jahrgang 2020: 159 159-172, 2020.

Rüfenacht, S., Woods, T., Agnello, G., Gold, M., Hummer, P., Land-Zandastra, A. and Sieber, A.: Communication and Dissemination in Citizen Science. In: Vohland K. et al. (eds) The Science of Citizen Science. Springer, Cham, https://doi.org/10.1007/9783-030-58278-4_24, 2021.

West, S. and Paeman, R.: Recruiting and Retaining Participants in Citizen Science: What Can Be Learned from the Volunteering Literature, https://doi.org/10.5334/cstp.8, 2016.

Wiggins, A. and Crowston, K.: From Conservation to Crowdsourcing: A Typology of Citizen Science. 2011 44th Hawaii International Conference on System Sciences, https://doi.org/10.1109/HICSS.2011.207, 2011.

Worthington, J. P., Silvertown, J., Cook, L., Cameron, R., Dodd, M., Greenwood, R.M., McConway, K. and Skelton, P.,: Evolution MegaLab: A case study in citizen science methods. Methods in Ecology and
Evolution, 3: 303-309, https://doi.org/10.1111/j.2041210X.2011.00164.X, 2011.

Ziello, C., Sparks, T., Estrella, N., Belmonte, J., Bergmann, K., Bucher, E., Brighetti, M., Damialis, A., Detandt, M., Galán, C., Gehrig, R., Grewling, Ł., Adela M., Gutiérrez-Bustillo, Hallsdóttir, M., KockhansBieda, M., De Linares, C., Myszkowska, D., Paldy, A., Sanchez, A., Menzel, A.: Changes to Airborne Pollen Counts across Europe. PloS one. 7. e34076. https://doi.org/10.1371/journal.pone.0034076, 2012. 